\title{
Integration of Postcoordination Content into a Clinical Interface Terminology to Support Administrative Coding
}

\author{
Eric Rose ${ }^{1,2}$ Steven Rube ${ }^{1} \quad$ Andrew S. Kanter ${ }^{1,3}$ Matthew Cardwell ${ }^{1} \quad$ Frank Naeymi-Rad ${ }^{1}$
}

1 Intelligent Medical Objects, Northbrook, Illinois, United States
2 Department of Biomedical Informatics and Medical Education,
University of Washington, Seattle, Washington, United States
${ }^{3}$ Department of Biomedical Informatics and Epidemiology, Columbia
University, New York, New York, United States

\author{
Address for correspondence Eric Rose, MD, Department of \\ Biomedical Informatics and Medical Education, University of \\ Washington, 4464 Fremont Avenue North, Seattle, WA 98103, \\ United States (e-mail: emrdoc@hotmail.com).
}

Appl Clin Inform 2019;10:51-59.

\section{Abstract}

Background Clinical interface terminologies (CITs) consist of terms designed for clinical documentation and, through mappings to standardized vocabularies, to support secondary uses of patient data, including clinical decision support, quality measurement, and billing for health care services. The latter purpose requires maps to administrative coding systems, such as the International Classification of Diseases, 10th Revision, Clinical Modification (ICD-10-CM), for diagnoses in the United States. Objectives The transition from ICD-9-CM to ICD-10-CM posed a challenge to CIT users due to the substantially increased details in ICD-10-CM. To address this, we developed a content layer within a CIT that provides postcoordination prompts for the details required for accurate ICD-10-CM coding.

Methods We developed content to support prompting for and capture of additional information specified by the user in a single, clinically relevant term that is added to the patient's record, and whose mapping to other coding systems (like Systematized Nomenclature of Medicine-Clinical Terms [SNOMED CT]) reflects the details added during postcoordination. We worked with clinical information system developers to incorporate this into user interfaces, and with end-users to refine the design.

Results While the prompts were designed around the precoordinated elements implicit in ICD-10-CM, irregularities in ICD-10-CM required some additional design measures, such as providing postcoordination options that interpolate gaps in ICD-10$\mathrm{CM}$ to avoid user confusion. The system we describe has been implemented by $\sim 30,000$ health care provider organizations, with content that covers the vast majority of encounter diagnoses. User feedback has been largely positive, though concerns have been raised about expanding postcoordination content beyond that required for ICD10-CM coding.

Conclusion We have demonstrated the design and development of what, to our knowledge, is the first system that uses postcoordination to capture ICD-10-CMrelevant details in a CIT while also reflecting the details added by the user in maps to other vocabularies. received

October 11, 2018

accepted after revision

November 22, 2018
DOI https://doi.org/

10.1055/s-0038-1676972.

ISSN 1869-0327. (c) 2019 Georg Thieme Verlag KG

Stuttgart · New York
License terms

(1)(1) $\Theta \circledast$ 


\section{Background and Significance}

A clinical interface terminology (CIT) is a set of terms designed to be used in a clinical information system (CIS) for clinical documentation at the point of care. CIT terms consist of words and phrases suited for populating structured data elements in a CIS, such as a patient's problem list, diagnoses for a patient encounter, the procedures that a patient has undergone in the past, or a patient's family medical history. In addition to terms, CIT content typically includes links (known as "maps") from individual terms to codes from standardized health vocabularies. These maps support secondary use of patient data, such as clinical decision support, quality measurement, interoperability, and billing for health care services. ${ }^{1,2}$ CITs serve as a "bridge" between CIS users and standardized health vocabularies by supporting variations in phrasing (lexical variation) as well as including terms whose meanings are more specific than codes in a standardized vocabulary (semantic extension). Also, by mapping a single term to codes from multiple standardized vocabularies, CITs reduce the need for duplicate data entry to support use cases that require codes from different vocabularies.

CITs support billing for health care services through maps to administrative coding systems. In the United States, the International Classification of Diseases, 10th Revision, Clinical Modification (ICD-10-CM) has been required for encoding diagnoses by most payers since October 1, 2015, replacing the diagnostic coding system ICD-9-CM, which had been required since the 1970 s. ${ }^{3,4}$ ICD-10-CM contains $\sim 70,000$ codes, as opposed to $\sim 14,000$ in ICD-9-CM. ${ }^{5}$ While the semantic scopes of ICD-10-CM and ICD-9-CM are the same (medical diagnoses), ICD-10-CM adds specificity in numerous semantic dimensions, including laterality, episode of care (e.g., initial encounter vs. subsequent encounter for a particular condition), the presence of specific complications of a chronic illness, and many other details. ${ }^{6}$

\section{Objectives}

For CIT users, this increase in coding details poses a challenge, since it greatly increases the specificity at which diagnoses must be expressed, and thus, the potential number of terms from which a user might have to choose. For example, for closed fracture of the wrist, ICD-9-CM includes 9 codes, while ICD-10$\mathrm{CM}$ has 75. ICD-9-CM provides one code for each wrist bone. ICD-10-CM, in addition to differentiating among fractures of different bones, provides separate codes for various combinations of other parameters, including whether the fracture is displaced or not, which wrist (left or right) is fractured, episode of care, and for some wrist bones, the particular subportion of the bone where the fracture is located (for instance, for the hamate bone, whether the fracture is located in the body or hook process of the bone). ICD-10-CM does, in some cases, provide codes that omit certain details, such as codes for fracture of "unspecified wrist" (i.e., not specifying whether the fracture is of the left or right wrist). However, use of those codes is discouraged if the additional information is known, and may result in less-favorable payment for clinical services. ${ }^{7}$
J45.2 Mild intermittent asthma

J45.20 Mild intermittent asthma, uncomplicated Mild intermittent asthma NOS

J45.21 Mild intermittent asthma with (acute) exacerbation

J45.22 Mild intermittent asthma with status asthmaticus

J45.3 Mild persistent asthma

J45.30 Mild persistent asthma, uncomplicated Mild persistent asthma NOS

J45.31 Mild persistent asthma with (acute) exacerbation

J45.32 Mild persistent asthma with status asthmaticus

J45.4 Moderate persistent asthma

J45.40 Moderate persistent asthma, uncomplicated Moderate persistent asthma NOS

J45.41 Moderate persistent asthma with (acute) exacerbation

J45.42 Moderate persistent asthma with status asthmaticus

J45.5 Severe persistent asthma

J45.50 Severe persistent asthma, uncomplicated Severe persistent asthma NOS

J45.51 Severe persistent asthma with (acute) exacerbation

Fig. 1 Illustration of patternistic precoordination in ICD-10-CM.

While the volume of codes in ICD-10-CM is large compared with ICD-9-CM, the precoordination of details into ICD-10-CM codes often follows certain regular patterns within a particular diagnostic area. That is, in many cases codes exist that instantiate each possible combination of values for multiple parameters. For instance, with asthma, ICD-10-CM provides 12 codes, which instantiate each of four values for severity ("mild intermittent," "mild persistent," "moderate persistent," and "severe persistent") as well as each of three values for complication status ("uncomplicated," "with acute exacerbation," and "with status asthmaticus"; - Fig. 1).

In this article, we report on the design and development of a system that makes use of the patternistic precoordination of ICD-10-CM as the basis for a postcoordination workflow that prompts clinical end-users for the specific details ("modifiers") necessary to navigate from a less-specific to a more-specific CIT term that ensures correct ICD-10-CM coding.

\section{Methods}

\section{General Approach}

The system was built as an extension to Problem (IT), a commercial CIT (Intelligent Medical Objects, Northbrook, IL, United States), and delivered as part of that product. Problem (IT) is integrated into various commercial and noncommercial CISs in the United States and other countries. It consists of $\sim 500,000$ terms for health-related findings and diagnoses, each of which is mapped to various standardized health vocabularies including ICD-9-CM, ICD-10-CM, and Systematized Nomenclature of Medicine-Clinical Terms (SNOMED CT).

The general design approach was predicated on several assumptions. First, it was assumed that the capture of information for ICD-10-CM coding should occur within the clinician's workflow for using the CIT to document encounter diagnoses. In other words, a separate workflow should not be required to capture ICD-10-CM codes, such as review of 
provider notes by a professional coder. Second, minimizing the cognitive burden on the end-user and avoiding the need for clinicians to become expert in ICD-10-CM were considered vital priorities. This ruled out approaches that might require the user to separately consult an ICD-10-CM reference book, or review lists of hundreds of terms or more. Lastly, to maximize the potential value of information captured from the clinician to support ICD-10-CM coding, the design had to ensure that such information would be incorporated into both the text of the CIT term added to patient's record and that term's maps to all standardized vocabularies (i.e., not only to ICD-10-CM, but also to other terminologies including SNOMED CT).

These assumptions, along with the observation of the patternistic nature of ICD-10-CM coding details mentioned above, led us to an approach that supplements the initial CIT search with targeted postcoordination prompts. In this approach, after the usual workflow of searching for and selecting a CIT term, the clinician is prompted with modifiers corresponding to the specific missing details required for ICD-10-CM coding for the selected term. These are grouped into one or more "modifier groups," each of which corresponds to a different semantic parameter. After selecting those modifiers that correspond to the patient's condition, the term initially selected is replaced with one that combines the initial term with the details added by the user, and that final term is added to the patient's record (-Fig. 2).

The content components of the system are shown in - Fig. 3 . Each CIT term that will trigger modifier prompts ("starting term"), and each CIT term to which the user can navigate by specifying modifiers ("ending term"), has a title for display in the CIS, a unique CIT identifier, and maps to one or more codes from multiple standardized vocabularies (a subset of which are shown in the figure), including, for the ending term, a billable ICD-10-CM code. In addition, each term is modeled with semantic tags ("attributes"). Some, but not all, attributes are designated as modifiers. If the modeling of two CIT terms is identical except for one or more modifiers, then the application logic will present them as modifier prompts when the starting term is selected. This content is stored in a relational database (Oracle Corporation, Redwood Shores, CA, United States) and managed using purpose-build software tools developed within our organization. The content was delivered both by flat database files and by a Web API for incorporation into CISs, whose developers coded the user interface layer in consultation with the organization that developed the content.

\section{Scoping and Content Build Process}

Several key scoping questions presented themselves early in the design process. The most significant of these was whether to attempt to provide modifier content covering the entirety of ICD-10-CM. ICD-10-CM includes numerous codes for which we could potentially have provided modifier options, but which are likely to be used infrequently, if at all, by the vast majority of clinicians. For example, there are 72 codes for injurious contact with birds, differentiating, among other parameters, injuries due to having been struck, pecked, or bitten, and injuries due to contact with a goose, duck, chicken, or turkey. We elected to focus content development on the most frequently diagnosed conditions in the United States. We utilized reports from the U.S. Healthcare Cost and Utilization Project, which collects data from a representative sample of hospitals in the United States, ${ }^{8}$ and from the American Academy of Family Physicians regarding common diagnoses for ambulatory encounters ${ }^{9}$ to develop a list of high-level diagnostic "concept areas." These became our initial scope for modifier content provided prior to the ICD-10-CM transition date of October 1, 2015. Since then, we have added modifier content for additional concept areas, selected based on input from users of the CIT as well as at the discretion of clinician members of the study team ( $\sim$ Fig. 4 ).

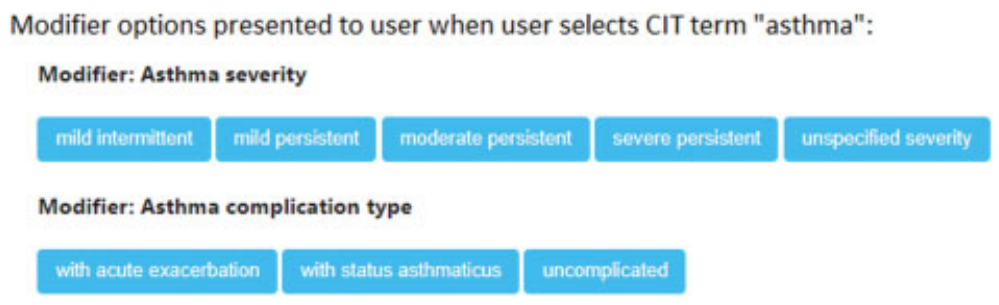
After user selects a modifier in each group, system returns a final term, whose meaning, code
maps, and internal CIT identifier incorporate the meaning of the starting term combined with
the selected modifiers.

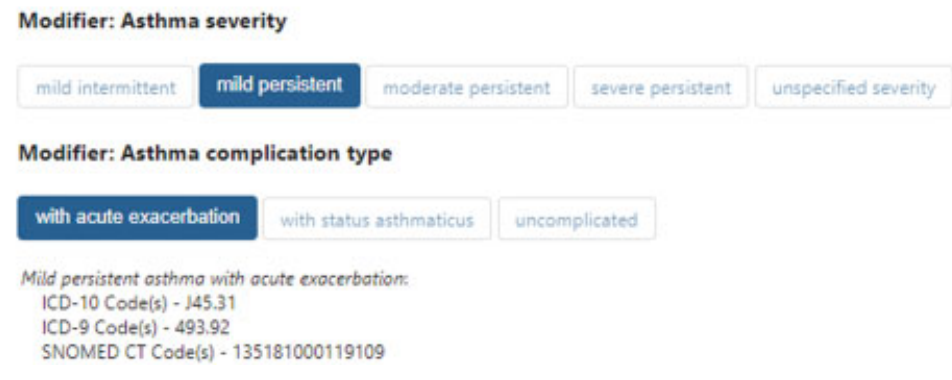

Fig. 2 Postcoordination user experience. 


\begin{tabular}{|c|c|c|c|}
\hline \multicolumn{2}{|l|}{$\begin{array}{l}\text { CIT term } \\
\text { CIT code: } 94262 \\
\text { CIT title: Asthma }\end{array}$} & $\begin{array}{l}\text { Vocabulary map } \\
\text { Vocabulary: ICD-10-CM } \\
\text { Code: } 145.909 \\
\text { Code title: Unspecified asthma, } \\
\text { uncomplicated }\end{array}$ & $\begin{array}{l}\text { Vocabulary map } \\
\text { Vocabulary: SNOMED CT } \\
\text { Code: } 195967001 \\
\text { Code title: Asthma (disorder) }\end{array}$ \\
\hline \multicolumn{4}{|l|}{$\begin{array}{l}\text { Attribute } \\
\text { Attribute ID: } 352705875 \\
\text { Attribute title: asthma } \\
\text { Is modifier: No } \\
\text { Modifier group: n/a }\end{array}$} \\
\hline \multicolumn{2}{|c|}{$\begin{array}{l}\text { CIT term } \\
\text { CIT code: } 1587651 \\
\text { CIT title: Mild persistent asthma with acute exacerbation }\end{array}$} & $\begin{array}{l}\text { Vocabulary map } \\
\text { Vocabulary: ICD-10-CM } \\
\text { Code: J45.31 } \\
\text { Code title: Mild persistent asthma } \\
\text { with (acute) exacerbation }\end{array}$ & $\begin{array}{l}\text { Vocabulary map } \\
\text { Vocabulary: SNOMED CT } \\
\text { Code: } 135181000119109 \\
\text { Code title: Acute exacerbation of mild } \\
\text { persistent asthma (disorder) }\end{array}$ \\
\hline $\begin{array}{l}\text { Attribute } \\
\text { Attribute ID: } 352705875 \\
\text { Attribute title: asthma } \\
\text { Is modifier: No } \\
\text { Modifier group: } n / a\end{array}$ & $\begin{array}{l}\text { Attribute } \\
\text { Attribute ID: } 1045 \\
\text { Attribute title: mild persistent asthma } \\
\text { Is modifier: Yes } \\
\text { Modifier group: Asthma severity }\end{array}$ & \multicolumn{2}{|c|}{$\begin{array}{l}\text { Attribute } \\
\text { Attribute ID: } 1042 \\
\text { Attribute title: asthma with acute exacerbation } \\
\text { Is modifier: Yes } \\
\text { Modifier group: Asthma complication type }\end{array}$} \\
\hline
\end{tabular}

Fig. 3 Content architecture.

\begin{tabular}{|ll|}
\hline Acne & Hypertension \\
Myocardial infarction & Migraine \\
Deep vein thrombosis & Oligomenorrhea \\
Dementia & Opioid dependence \\
Diverticulitis & Pancreatitis \\
Epilepsy & Pneumonia \\
Fever & Rotator cuff tear \\
Fracture & Sepsis \\
Gastrointestinal hemorrhage & Syncope \\
Gout & Viral hepatitis \\
\hline
\end{tabular}

Fig. 4 Partial list of diagnostic categories covered.

Another key scope decision was whether to include modifier options within a concept area beyond those strictly required for ICD-10-CM coding, where they might have clinical value. For example, ICD-10-CM differentiates codes for pneumonia based on causal organism or other etiologic factor, but not based on location, i.e., which lung and/or lobe(s) of the lung is involved. However, it is generally considered of clinical value to record the location of pneumonia when it is known. Recognizing a need to balance the value of such additional detail with the burden imposed on the clinician by presenting additional modifier options, we took a parsimonious approach to extending modifier options beyond those required for ICD10-CM coding. We consulted frequently with CIT users on specific design decisions and added them only when (as was the case with pneumonia location) there was a strong consensus that the value of capturing the additional details outweighed the burden of additional modifiers.

We developed a structured content build process, which was performed by a multidisciplinary team that included clinician informaticists, nonclinician terminologists, and certified professional coders (CPCs). The content build process involved the following steps for each concept area:
Step 1: Define the concept area. This step consisted of identifying and naming the concept area, and compiling a list of the ICD-10-CM codes to which it corresponds. As noted above, the initial set of concept areas were derived from published sources describing frequently diagnosed conditions in inpatient and outpatient care in the United States. We utilized several techniques to identify the codes for each concept area. First, we manually reviewed the hierarchical sections of ICD-10-CM to identify areas where relevant codes might exist. Next, to identify codes that might not have been found on the section review, we performed computerized text searching of published electronic versions of the ICD-10$\mathrm{CM}$ code titles as well as the ICD-10-CM indexing guide. ${ }^{10} \mathrm{We}$ also consulted with CPCs on our content development team to identify cases where coding guidelines require use of an ICD-10-CM code for a particular diagnosis even though the literal meaning of the ICD-10-CM code title does not correspond to that diagnosis. As a result, we frequently found that the ICD-10-CM codes corresponding to a concept area were located in multiple noncontiguous sets of codes. For example, as shown in -Fig. 5, for the concept area "syncope," relevant ICD-10-CM codes include codes from three different sections of ICD-10-CM (R55, "Syncope and collapse," G90.01, "Carotid sinus syncope," and T67.1XXA/D/S, "Heat syncope"), as well as codes that did not include any mention of "syncope" in the code title, but were nonetheless appropriate codes for clinically relevant subtypes of syncope. In other cases, while the one set of ICD-10-CM codes might be appropriate for primary coding of a diagnosis, ICD-10-CM coding guidelines require submission of a second code to describe some aspect of the condition. For example, with anemia due to chronic kidney disease (D63.1), ICD-10-CM requires an additional code to identify the stage of chronic kidney disease (N18.1-N18.9). In such cases, we included the required secondary codes among the ICD-10-CM codes for a concept area. 


\begin{tabular}{|l|l|l|}
\hline CIT term & ICD-10-CM code(s) & ICD-10-CM code title \\
\hline Carotid sinus syncope & G90.01 & Carotid sinus syncope \\
\hline Heat syncope & T67.1XXA, T67.1XXS, T67.1XXD & Heat syncope \\
\hline Psychogenic syncope & F48.8 & Other specified nonpsychotic mental disorders \\
\hline Stokes-Adams syncope & 145.9 & Conduction disorder, unspecified \\
\hline Vasovagal syncope & R55 & Syncope and collapse \\
\hline
\end{tabular}

Fig. 5 Example of discontinuous ICD-10-CM codes required for a single concept area.

Step 2: Determine the modifiers and modifier groups for postcoordination. In this step, clinician members of our team performed a human review of the codes and code titles to analyze the details implicit in the ICD-10-CM codes identified in Step 1 and determined what modifier groups should be presented to the end-user when they select a CIT term corresponding to the high-level concept area. A manual, human-designed approach was taken for this step because no automated approaches were found to be feasible. While there are some regular patterns within ICD-10-CM within a given concept area, each concept area has its own idiosyncratic patterns, so it was not possible to replicate modifier design across concept areas in an automated fashion. While SNOMED CT has a machine-computable composition from which hierarchical relationships and attribute names and values can be extracted, and which is intended to support postcoordination workflows, there is not close enough correspondence between SNOMED CT concepts and ICD-10-CM codes, nor between the semantic subclassification of concept areas between the two coding systems, to have used the former to automatically derive content needed to seamlessly navigate the latter.

Several careful design considerations were brought to bear in this step. For each concept area, one modifier group was created for each distinct, semantically orthogonal parameter implicit in the ICD-10-CM codes. For example, for the concept area "back pain," modifier groups were created for "location," "chronicity," "laterality," and "sciatica presence," since these were the parameters reflected in the ICD-10-CM codes for this concept area. We attempted, whenever possible, to ensure that modifiers within a particular modifier group described are mutually exclusive. This helps to avoid situations where more than one modifier within a group might accurately describe a patient's condition, since that could leave users confused as to which modifier to select. Also, we attempted to ensure that the modifiers within a group cover all possible clinical situations, so that users did not face situations where none of the available modifier options within a group were consistent with the patient's condition. For instance, for acute Streptococcal tonsillitis, ICD-10-CM offers a code for "Acute recurrent streptococcal tonsillitis" (J03.01) but no code for "Acute nonrecurrent streptococcal tonsillitis." To address this gap, our modifier group "Streptococcal tonsillitis recurrence" includes options for both "recurrent" and "nonrecurrent." The CIT term to which the "nonrecurrent" modifier option leads is not mapped to an ICD-10-CM code that reflects that information (per ICD-10-CM guidelines it is mapped to J03.00, "Acute streptococcal tonsillitis, unspecified"), but inclusion of the "nonrecurrent" modifier helps the user avoid confusion resulting from the asymmetric nature of the available ICD$10-\mathrm{CM}$ codes in this concept area. Lastly, we determined the specific order in which modifiers would be listed in the user interface within modifier groups, and the overall ordering of modifier groups. This could have significant impact on the user experience, particularly in concept areas for which a large number of modifiers and/or modifier groups were required. In some cases, we determined (often based on user feedback) that one modifier was likely to be selected much more frequently than others in the same modifier group, and in those cases, we elected to place it before other modifiers in the group. An example of this, shown in -Fig. 6A, is the modifier "iron deficiency" within the modifier group "Anemia type," which has six other modifiers. In cases where a modifier list is lengthy and no one modifier within the group is likely to be selected much more frequently than others, we followed alphabetical ordering to allow for easier visual scanning (see - Fig. 6B).

Step 3: Ensure that the CIT includes terms needed for the concept area. In this step, we determined which terms in the CIT would be used as the starting and ending terms for postcoordination for the concept area. This included three types of terms.

- "Root terms," which correspond to the concept area at its highest level and serve as starting terms. These generally matched the name of the concept area as well as various lexical variants (e.g., for concept area "ankle pain," the root terms included "ankle pain," "pain in ankle," "painful ankle," and "pain, ankle").

- "Ending terms," which correspond to the end-points of postcoordination (e.g., "chronic pain of left ankle").

- "Intermediate terms," which instantiate the meaning of the root term and modifiers from some subset of the modifier groups for the concept area (e.g., "chronic ankle pain" or "pain of left ankle"). These terms ensure that if an end-user happens to select a term that instantiates some, but not all, of the details required by ICD-10-CM, they are prompted for any remaining necessary details to arrive at an ending term.

In most cases, the list of terms needed in this step was already included in the CIT. Where necessary, this step included creation of new CIT terms and mapping of those 
Modifier: Anemia type

\begin{tabular}{|c|c|c|c|c|c|c|}
\hline iron defliciency & B12 deficiency & folate deficiency & bone marrow fallure & acquired or hereditary hemolytic anemia & due to chronic kidney disease & other cause \\
\hline
\end{tabular}

A

Modifier: Internal fixation site

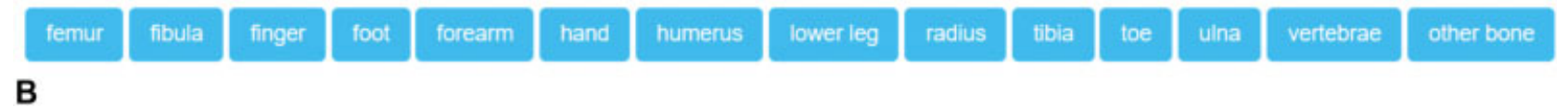

Fig. 6 (A, B) Design considerations with ordering of modifiers within a modifier group.

terms to standardized vocabularies, to ensure that the CIT included all terms needed for the concept area.

Step 4: Model the CIT terms with attributes. As described above and in -Fig. 4, the postcoordination experience is driven by tagging of CIT terms with "attributes" that reflect the meaning of the term. In this step, attributes are assigned to each of the terms identified in Step 3. Each term is assigned the attribute(s) of the root term for the concept area, and also those modifier attributes that reflect the difference between that term and the root term.

Step 5: Testing. We created a simulator application that automatically derives the modifier differences between starting, intermediate, and ending terms, and used that to test the results of steps 1 to 4 to make sure that all appropriate modifiers were displayed, that they were grouped and ordered correctly, that all appropriate (and no extraneous) postcoordination combinations were available, and that the ending terms were correctly phrased and mapped.

Step 6: Release. Once testing was completed for a concept area, the modifier content for that concept area was integrated into the next release of the CIT, for incorporation into the CISs that utilized this content. With each release, organizations using the CIT received detailed documentation of any new modifier content, including the details of modifier groups, modifiers, and ICD-10-CM codes covered by each new concept area.

User acceptance testing and feedback: since each concept area presented its own unique design challenges, user acceptance testing was conducted at the level of the concept area. Clinicians from both within and outside the developing organization were consulted regarding the design of each concept area, including both mock-ups and a live application that simulated the experience within the CIS. In addition, feedback from a larger group of users was sought after the release of modifier content for each concept area. Based on this feedback, adjustments were sometimes made to the modifier design for a concept area, e.g., in the ordering of modifiers or modifier groups or in the overall scope of a concept area (e.g., lumping two related concept areas together under a more coarsely granular root term).

\section{Design Challenges}

As content build work proceeded, several design challenges emerged. We found that designing the postcoordination user experience to rigidly match the available ICD-10-CM codes would likely be confusing and dissatisfying for end-users. Fortunately, our content architecture did not rigidly bind either our CIT terms nor the attribute modeling to ICD-10-CM codes or titles (see - Fig. 3). As a result, we were able to design the content to mitigate the irregularities of ICD-10-CM, and provide what we anticipated to be a more intuitive user experience.

Interpolating gaps in ICD-10-CM: in many cases, ICD-10$\mathrm{CM}$ does not provide separate codes for all clinically valid combinations of the parameters expressed in a code range. For instance, the codes for chronic ischemic heart disease (I25) include variations on the type of artery affected (e.g., native artery, bypass graft, etc.) and whether and what type of angina pectoris is present. There are codes for atherosclerosis with angina pectoris affecting a native coronary artery (I25.11), an autologous vein bypass graft (I25.71), an autologous artery bypass graft (I25.72), and a nonautologous biological bypass graft (I25.73). For atherosclerosis without angina pectoris, there are codes specifying involvement of a native coronary artery (I25.10) and a bypass graft generally (I25.810), but none for the aforementioned three subtypes of bypass graft. To avoid a user experience in which some combinations of modifier options might be unavailable for no reason that would be apparent to the end-user, we provided CIT terms for all clinically valid combinations of these parameters, and allow users to specify the corresponding combinations of modifiers, even though the result is that multiple ending terms have the same ICD-10-CM code map. Similarly, in many cases, ICD-10-CM provides codes for "left" and "right" versions of a condition involving a paired body part, but not a "bilateral" option; where clinically valid, we have generally included one (see - Fig. 7).

Clinically awkward categorization in ICD-10-CM: we found that in some cases, ICD-10-CM's approach to subdividing a concept area did not seem clinically intuitive. Since ICD-10-CM is a classification intended for administrative and statistical use and thus not designed specifically to facilitate clinically intuitive documentation, ${ }^{3}$ these issues present challenges when attempting to facilitate capture of data at the point of care to support ICD-10-CM coding. In such cases, we undertook to provide modifier options that semantically split the concept area more finely than ICD-10-CM in ways that were more clinically logical. For example, ICD-10-CM codes for gout instantiate the affected joint. In most cases, separate codes are provided for individual joints, but for the "ankle and foot," no differentiation is provided. Our approach, rather than provide 


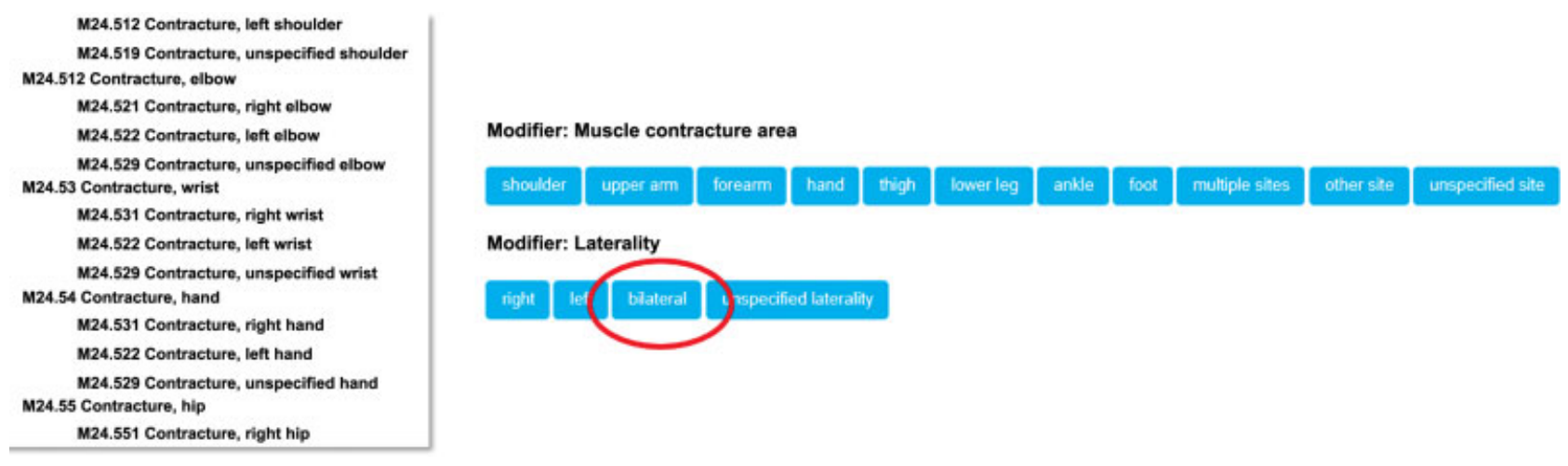

Fig. 7 Interpolating gaps in ICD-10-CM.

a modifier option of "ankle and foot" for end-users to specify the site of gout, was to provide two separate modifiers, one for "ankle" and one for "foot." Each option results in an ending term that specifies the single joint named in the modifier, though the corresponding ICD-10-CM code does not differ between them (see - Fig. 8).

Adjusting ICD-10-CM verbiage to maintain editorial conformance in the CIT: ICD-10-CM code titles often do not conform to the editorial standards that are in place to ensure validity and usability in the CIT. Since CIT terms are maintained as a separate data object from the titles of the ICD-10-CM codes to which they are mapped (see - Fig. 3), we were able, where necessary, to maintain conformance to those editorial standards by choosing verbiage for CIT terms that differs from the precise wording of ICD-10-CM code titles. These editorial standards are intended to achieve the following goals in the CIT.

- Maintenance of consistency, e.g., the use of "upper extremity" in the CIT instead of the ICD-10-CM convention of "upper limb."

- Avoidance of redundancy, e.g., the use of "eyelid" instead of "eyelid, including canthus" as used in some ICD-10-CM code titles such as D22.1.

- Maintenance of clarity, e.g., the use of "gastric cardia" rather than simply "cardia" as used in some ICD-10-CM code titles, such as C16.0.

- Adherence to common clinical conventions, e.g., the use of "lipoma" instead of "benign lipomatous neoplasm" as used in some ICD-10-CM code titles, such as D17.0.

\section{Results}

The initial release of modifier content, for the initial ICD-10-CM transition in October 2015, covered $\sim 300$ concept areas, corresponding to at least $95 \%$ of primary encounter diagnoses for ambulatory and hospital encounters in the United States. ${ }^{7,8}$ For this initial set of content, the design phase (steps 1 and 2 as described in the "Scoping and content build process" section above) was performed by two clinician informaticists largely over a 3-week period, and the build phase (steps 3-5) by the larger multidisciplinary team over 12 months. Since then, we have added additional content and as of the time of publication, have released modifier content over 420 concept areas. The concept areas range from only a few terms to over 3,000 terms in some instances.

This content has been implemented in clinical documentation workflows by $\sim 30,000$ health care provider organizations in the United States, ranging from solo physician practices to multihospital academic medical centers, including over 2,000 hospitals.

In addition to the release of new concept areas, revisions to existing modifier concept areas have been needed as a result of annual updates to ICD-10-CM. For instance, in the 2017 update of ICD-10-CM, new codes were added for diabetic retinopathy specifying laterality and, for proliferative retinopathy, four specific subtypes. Accordingly, we added terms and modifier content to ensure that users could navigate to CIT terms mapped to those new ICD-10-CM codes.

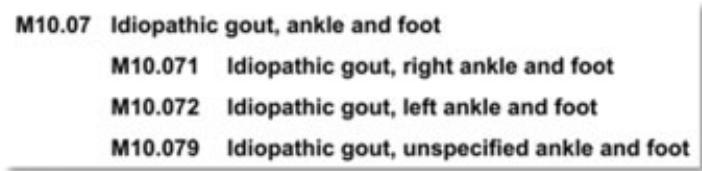

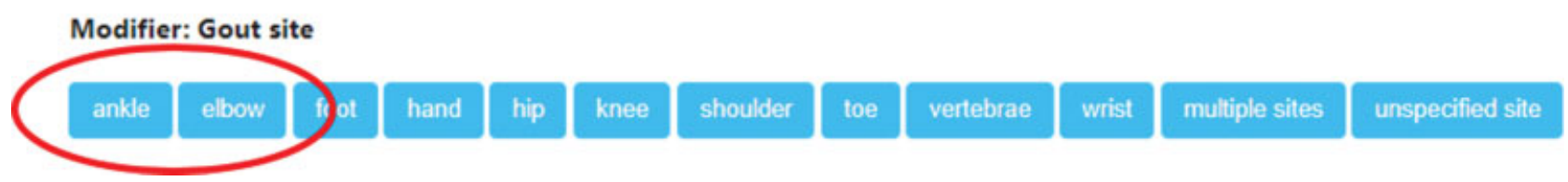

Fig. 8 Mitigating clinically awkward categorization in ICD-10-CM. 
As mentioned above, our content scoping and build processes incorporated feedback from end-users. Some organizations have expressed a strong desire for expansion of modifier content. These requests have tended to be focused primarily on content that could be relevant for ICD-10-CM coding (R. Leviton, personal communication, November 2014). In some cases, specialty-focused organizations requested expansion of modifier content covering additional concept areas relevant to a particular specialty (S. Magid, personal communication, October 2015). We have utilized this feedback to guide the choices of content we have added since October 2015.

Some organizations have requested modifier content to capture clinical details beyond those necessary for optimal ICD-10-CM mapping, to facilitate data capture for quality reporting, clinical decision support, or clinical research (J. Moy, personal communication, May 2016). Others have expressed concern about the additional data entry burden that modifier content poses, and have advocated that such content should be limited to that directly relevant to ICD-10-CM coding (H. Yang, personal communication, September 2015).

\section{Discussion}

We have described the development of a content layer within a widely used commercial CIT that provides users, after selecting a term, with prompts to postcoordinate the details required to enable accurate documentation and appropriate ICD-10-CM coding. This approach captures the additional information specified by the user in a single, clinically relevant term that is added to the patient's record, and whose mapping to other coding systems (like SNOMED $\mathrm{CT}$ ) reflects those additional details. To our knowledge, this is the first system that uses postcoordination to capture ICD-10-CM-relevant details in a CIT while also reflecting the details added by the user in maps to other vocabularies. The content has been implemented in a large number of clinical practices.

By conceptually deconstructing the precoordination patterns in ICD-10-CM, we have replaced a workflow that might require a user to scan hundreds or thousands of terms with one in which the user is presented with a list of modifiers and simply clicks those that apply. This approach ensures that administrative codes are captured from the clinician at the point of care, rather than deduced after the fact by a nonclinician reviewing the medical record. In addition, it helps ensure textual and coded entries in the medical record (including SNOMED CT codes) that reflect information that in many cases may be of clinical relevance, such as whether a fracture is open or closed, or which coronary arteries were involved in a patient's myocardial infarction.

Because the architecture of our solution is not rigidly bound to the structure of ICD-10-CM, it allowed the postcoordination experience to be designed in ways that mitigated many of the aspects of ICD-10-CM that could confuse clinicians, such as cases where codes are not provided for all clinically relevant combinations of different parameters. This also made it simple to adapt the content to the addition of new ICD-10-CM codes in annual updates of the coding system since the initial release of our solution. This same flexibility provided the design team with some latitude to expand the postcoordination options beyond those strictly required for ICD-10-CM coding. We elected to do so sparingly, and the feedback we received from end-users of the system indicates the need to strike a delicate balance between adding postcoordination options that might help capture important details and avoiding imposing an excessive data-entry burden on clinicians.

For future development, we plan to continue to adjust the content to account for changes in annual updates of ICD$10-\mathrm{CM}$, as well as adding new concept areas. In addition, we are considering adding content to optimize coding for vocabularies other than ICD-10-CM. For instance, The Diagnostic and Statistical Manual of Mental Disorders, 5th Edition (DSM-5) provides a highly precoordinated list of terms to be used in the classification of mental illnesses, ${ }^{11}$ which could potentially benefit from the approach described in this article. In conjunction with this, we are exploring ways to tailor the implementation of the content by individual provider organization, allowing organizations to utilize only the content related to target vocabularies of interest.

Certain limitations of this study are worth noting. We have described the successful design and development of a novel approach for facilitating capture of highly detailed administrative diagnostic codes in clinical workflows. We did not, however, directly assess the impact of this approach on user satisfaction, efficiency of CIS use, provider revenue cycle, or patient outcomes. We believe these are potentially fruitful avenues for future research.

\section{Clinical Relevance Statement}

The need to capture the specific details required by administrative coding systems presents a serious challenge to clinicians documenting diagnoses in a CIS. While use of a CIT as the means to document diagnoses provides many advantages, the CIT terms initially selected by users may not include the details required for optimal administrative coding. This article describes one solution that addresses this issue while avoiding the need for individual clinicians to master the intricacies of administrative coding systems.

\section{Multiple Choice Questions}

1. What is a clinical interface terminology (CIT)?

a. A set of codes required to bill for medical services in the United States.

b. A set of terms designed for clinical documentation and to support secondary uses of patient data.

c. A set of cross-maps from one vocabulary (like SNOMED) to another (like ICD-10-CM).

d. The text found in documents in medical records, such as physician notes.

Correct Answer: The correct answer is option b. 
2. How many more codes are in ICD-10-CM compared with ICD-9-CM?

a. Twice as many.

b. Three times as many.

c. Five times as many.

d. Ten times as many.

Correct Answer: The correct answer is option c.

3. Which of the following presented a design challenge in creating postcoordination prompts to ensure capture of ICD-10-CM-relevant details?

a. ICD-10-CM does not provide codes for common diagnostic concepts.

b. ICD-10-CM has multiple codes with the exact same meaning.

c. Updates to ICD-10-CM are not published until they go into effect.

d. In some cases, the way that ICD-10-CM subdivides a diagnostic area may not be intuitive to clinicians.

Correct Answer: The correct answer is option d.

\section{Protection of Human and Animal Subjects}

Human and/or animal subjects were not included in the work described in this article.

\section{Conflict of Interest}

All of the authors are employees of Intelligent Medical Objects, a commercial vendor of terminology solutions, which developed the system described in this article.

\section{References}

1 Rosenbloom ST, Miller RA, Johnson KB, Elkin PL, Brown SH. Interface terminologies: facilitating direct entry of clinical data into electronic health record systems. J Am Med Inform Assoc 2006;13(03):277-288

2 Naeymi-Rad F. A Feature Dictionary to Support Database Translation, Information Retrieval, Intelligent Medical Records, and Expert Systems [PhD Dissertation]. Chicago, IL: Illinois Institute of Technology; 1990

3 Steindel SJ. International classification of diseases, 10th edition, clinical modification and procedure coding system: descriptive overview of the next generation HIPAA code sets. J Am Med Inform Assoc 2010;17:274-282

4 Conn J. ICD-10 one year later: The drama is over, the rewards yet to materialize. Mod Healthc 2016;46(41):10

5 National Center for Health Statistics. International Classification of Diseases, (ICD-10-CM/PCS) Transition - Background. Available at: https://www.cdc.gov/nchs/icd/icd10cm_pcs_background.htm. Accessed February 6, 2018

6 National Center for Health Statistics. ICD-10-CM Tabular List of Diseases and Injuries, 2018 ed. Washington, DC2017

7 Georgia Department of Community Health. ICD-10 Frequently Asked Questions. Available at: https://dch.georgia.gov/sites/dch. georgia.gov/files/ICD-10\%20FAQ\%20FINAL\%2020151103.pdf. Accessed February 17, 2018

8 Agency for Healthcare Research and Quality. Healthcare Cost and Utilization Project. Available at: https://www.ahrq.gov/research/ data/hcup/index.html. Accessed February 17, 2018

9 Spencer DS, Whitecar PS, Daugird A. ICD-9 Codes for Family Medicine 2011-2012: the FPM Short List. American Academy of Family Physicians, 2011. Available at: http://www.aafp.org/journals/fpm/explore/topic/icd-9.html. Accessed November 8, 2014

10 National Center for Health Statistics. ICD-10-CM Index to Diseases and Injuries, 2018 ed. Washington, DC2017

11 American Psychiatric Association. Diagnostic and statistical manual of mental disorders, 5th ed. Washington, DC; 2013 